\title{
Intramuscular 3-Hydroxybutyrate Levels after 60 Tetani/Min Contraction in Diabetic and Non-Diabetic Rats
}

\author{
YoshiHARU OSHIDA, JUICHI SATO, NAOYA NAKAI, YoshiHARU SHIMOMURA*, \\ ISAO OHSAWA, AND YUZO SATO \\ Research Center of Health, Physical Fitness and Sports, Nagoya University, Nagoya 464-8601, and \\ *Nagoya Institute of Technology, Nagoya 466-8555, Japan
}

\begin{abstract}
Post-exercise hyperketonemia in poorly-controlled diabetic patients is a well recognized phenomenon, but because studies concerning changes in ketone body metabolism in muscle during and/or after exercise are scarce, we measured the intramuscular 3-hydroxybutyrate (3-OHBA) and lactate concentrations in 12 diabetic (streptozotocin-induced; $60 \mathrm{mg} / \mathrm{kg}$, ip) and 13 non-diabetic control rats before and after a $1-\mathrm{h}$ muscle contraction. One thigh tetanic contraction was elicited at a tetanic frequency of 60 tetani/min and the other thigh was kept resting. We used a microdialysis technique in both quadriceps muscles, vastus lateralis, and the right jugular vein. Blood flow in both femoral muscles was also monitored before and after contraction. Dialysate 3-OHBA and lactate levels in contracting and non-contracting muscles and in blood showed a significant increase after contraction $(P<0.05)$ in diabetic rats. In control rats there was no significant change in dialysate 3-OHBA or the lactate concentration in contracting and non-contracting muscles or in blood before and after contraction. A significant increase in contracting muscle blood flow was observed only for the first $5 \mathrm{~min}$ after contraction in diabetic and control rats. These results suggest that 3-OHBA uptake in contracting muscle is reduced, and that this phenomenon may play a role in post-exercise ketosis in diabetes.
\end{abstract}

Key words: 3-Hydroxybutyrate, Microdialysis, Muscle contraction, Diabetes

(Endocrine Journal 45: 363-370, 1998)

THE beneficial effects of physical exercise training have been well established in healthy people [1] and include an improvement in carbohydrate metabolism in glucose-intolerant subjects $[2,3]$. The metabolic response to exercise in Type 1 diabetic patients depends on a variety of factors. Especially, the level of glycemic control and the time elapsed since the last insulin injection play essential roles. When exercise is performed by a patient in good metabolic control immediately after a subcutaneous insulin injection, there is a nearly normal rise in free fatty acids (FFA) and a fall in blood glucose,

Received: October 13, 1997

Accepted: March 4, 1998

Correspondence to: Dr. Yoshiharu OSHIDA, Research Center of Health, Physical Fitness and Sports, Nagoya University, Furo-cho, Chikusa-ku, Nagoya 464-8601, Japan which can often lead to severe hypoglycemia [4]. On the other hand, in ketotic hyperglycemic insulindeprived diabetic patients [5], exercise may provoke a further increase in blood glucose and ketone body concentrations.

It is well known that the post-exercise hyperketonemia in diabetes results from an exaggerated stimulation of ketogenesis [5, 6], but the progression of ketosis may be due not only to increased production in the liver but also reduction in peripheral utilization $[7,8]$. Studies concerning changes in muscle ketone body metabolism during and/or after exercise are few and provide conflicting data. Féry et al. [9] demonstrated that the hyperketonemic effect of prolonged exercise in ketotic diabetics was caused by a defect in the capacity for removal of ketones. On the other hand, Wahren et al. [6] found an increase in ketone body 
utilization by muscle during exercise, and little attention has been paid to changes in the intramuscular ketone body level before and after exercise.

We recently reported that the microdialysis technique can be used to measure intramuscular 3-hydroxybutyrate (3-OHBA) [10] and lactate levels [11]. With this technique, therefore, the present study was undertaken to clarify the effect of tetanic contraction (60 tetani/min) on intramuscular 3OHBA and lactate levels in diabetic and non-diabetic control rats.

\section{Materials and Methods}

\section{Materials}

Twenty-five male Wistar rats (250-280 g B.W., Chubu Kagakushizai Co., Nagoya, Japan) were housed in an air-conditioned laboratory room with a 12-h light-dark cycle. The animals had free access to laboratory chow and water. Diabetes was induced by the intraperitoneal administration of streptozotocin (STZ, Sigma Chemical Co., USA) at a dose of $60 \mathrm{mg} / \mathrm{kg}$. A solution of STZ was freshly prepared in buffered citrate, $\mathrm{PH}$ 4.0. Twelve rats were given STZ (diabetic) and then placed in individual cages for 7 days until the start of the experiment. The other rats were injected with vehicle alone (non-diabetic control).

\section{Experimental protocol}

All were anesthetized with intraperitoneal pentobarbital sodium $(40 \mathrm{mg} / \mathrm{kg})$. A rectal thermometer was introduced and the rectal temperature was kept at $37.0^{\circ} \mathrm{C}$ by adjusting the distance from a heating lamp. As previously reported $[10,11]$, microdialysis probes, which consisted of a tubular membrane (polycarbonate, outer diameter $0.5 \mathrm{~mm}$, length $10 \mathrm{~mm}$, molecular weight cut-off 5,000-20,000 daltons, Carnegie Medicin, Sweden), were inserted bilaterally into the quadriceps muscles, vastus lateralis, and were also put into the right jugular vein by using the guide for the blood microdialysis. Probes were perfused at $1.4 \mu \mathrm{l} / \mathrm{min}$ with Krebs-Henseleit buffer (KHB), PH 7.4, and then the dialysate was collected at 30-min intervals for $60 \mathrm{~min}$ before contraction and for $90 \mathrm{~min}$ after contraction after a $60 \mathrm{~min}$ stabilization period. Both ends of the femoral muscle were exposed via an incision in one thigh for connecting to electrodes. Tetanic contraction of one thigh was elicited with supramaximal square-wave pulses $(6 \mathrm{~V}, 0.05 \mathrm{~ms})$ at a tetanic frequency of 60 tetani/min (100 ms train, $100 \mathrm{~Hz})$ [12]. The other thigh was kept resting throughout the study. The treatment was stopped after 60 $\mathrm{min}$, and then samples were collected every $30 \mathrm{~min}$ for $90 \mathrm{~min}$. Blood flow in contracting and noncontracting muscles was measured before and after contraction by means of laser blood flow meter (LBF-III; Biomedical Science, Kanazawa, Japan). During contraction, microdialysis sampling or blood flow monitoring was not done because probes of the microdialysis and the blood flow meter were easily moved.

\section{In vivo calibration}

The true concentrations of 3-OHBA and lactate in the intramuscular space were determined by the equilibrium technique [13] in 5 STZ-induced diabetic and 5 non-diabetic control rats. In brief, one femoral muscle was perfused with $\mathrm{KHB}$ containing different concentrations of 3-OHBA and the other was perfused with $\mathrm{KHB}$ containing different concentrations of lactate at the rate of 1.4 $\mu \mathrm{l} / \mathrm{min}$. At each concentration, there was a $60-$ min perfusion and samples were collected at $30-\mathrm{min}$ intervals. 3-OHBA and lactate concentrations in the perfusate and dialysate were measured, and the true concentrations of these metabolites in the intramuscular space were determined.

\section{Analytical methods}

3-OHBA and lactate in muscle and jugular vein dialysates were measured every $30 \mathrm{~min}$ by means of KETO-340 II (Sanwa Chemical Co., Japan) and a YSI lactate analyzer (Yellow Springs Instrument, USA), respectively. The in vitro recovery rates of 3-OHBA and lactate (dialysate concentration $v s$. medium concentration $\times 100$ ) were measured as previously reported $[10,14]$ and were then $60.8 \pm$ $3.4 \%$ and $56.6 \pm 1.1 \%$, respectively, at a perfusate flow rate of $1.4 \mu \mathrm{l} / \mathrm{min}$. Probes were placed in media containing varying concentrations of 3OHBA (20 to $800 \mu \mathrm{mol} / l$ ) and lactate (0.3 to 3.0 
$\mathrm{mmol} / l$ ) and were continuously perfused with $\mathrm{KHB}$ at $1.4 \mu \mathrm{l} / \mathrm{min}$. Dialysate concentrations of these substances were measured in triplicate at each concentration. The recovery remained constant at the 3-OHBA and lactate concentrations of up to at least $800 \mu \mathrm{mol} / l$ and $3.0 \mathrm{mmol} / l$, respectively. Plasma glucose, free fatty acids (FFA) and insulin concentrations were determined at the base line, and immediately and $90 \mathrm{~min}$ after contraction with a YSI glucose analyzer (Yellow Springs Instrument, USA), enzymatically and by radioimmunoassay (Phadeseph Insulin RIA, Pharmacia AB, Sweden), respectively.

All data were expressed as means $\pm \mathrm{SEM}$. Within each group, the statistical significance of the changes in 3-OHBA and lactate in dialysate was evaluated by the non-parametric Wilcoxon rank sum test. Comparison of plasma glucose, FFA and insulin concentrations in diabetic and control rats was performed by Student's $t$-test. A $P$ value less than 0.05 was considered significant.

\section{Results}

\section{Effects of tetanic contraction}

Before contraction, plasma glucose, FFA and insulin concentrations in diabetic rats were significantly different from those in controls, as shown in Table 1 . There was no significant difference in plasma glucose, FFA or insulin concentrations before and after tetanic contraction in both groups.

As shown in Fig.1, tetanic contraction resulted in a significant increase in 3-OHBA and lactate levels in jugular vein dialysate (from $295 \pm 24$ to $413 \pm 72 \mu \mathrm{mol} / l$, from $1.0 \pm 0.1$ to $1.2 \pm 0.1 \mathrm{mmol} / l$, respectively, $P<0.01$ ), which were observed for 60 $\min$ and for $30 \mathrm{~min}$, respectively, after contraction in diabetic rats. Blood dialysate 3-OHBA and lactate concentrations were kept constant in control rats. In contracting muscle dialysate (Fig. 2), 3OHBA concentrations in diabetic rats were significantly greater $90 \mathrm{~min}$ after contraction (310 $\pm 64 \mu \mathrm{mol} / l, P<0.05)$, than at the pre-contraction level $(211 \pm 16 \mu \mathrm{mol} / l)$. There was also a significant increase in the lactate concentration $30 \mathrm{~min}$ after contraction ( $1.0 \pm 0.1$ vs. $1.3 \pm 0.1 \mathrm{mmol} / l, P<0.05)$. In control rats, muscle contraction did not significantly increase 3-OHBA and lactate concentrations. In non-contracting muscle (Fig. 3), a significant increase in 3-OHBA concentrations in diabetic rats was found $60 \mathrm{~min}$ after tetanic contraction (from $201 \pm 22 \mu \mathrm{mol} / l$ to $342 \pm 65 \mu \mathrm{mol}$ / $l, P<0.05)$. There was no significant increase in the dialysate lactate concentration in diabetic rats after contraction. Dialysate 3-OHBA and lactate concentrations in control rats did not change significantly.

Fig. 4 shows blood flow monitoring in contracting and non-contracting muscles of diabetic (a) and control rats (b). A significant increase in contracting muscle blood flow was found for the first $5 \mathrm{~min}$ after contraction in both groups. Non-contracting muscle blood flow remained constant.

\section{The true intramuscular concentrations of 3-OHBA and lactate}

The relationship between the concentrations of two substrates in perfusate and dialysate during an equilibrium microdialysis in vivo is shown in Figs. 5 and 6 . When the perfusate concentration is the same as the intramuscular concentration, there will be no change in the dialysate concentration (i.e. equilibrium). This perfusate concentration is the true intramuscular concentration. The true 3-

Table 1. Plasma insulin, glucose and free fatty acid (FFA) concentrations at the base line, and immediately and 90 min after contraction

\begin{tabular}{|c|c|c|c|c|c|c|c|c|c|c|c|c|}
\hline & \multicolumn{4}{|c|}{ Insulin (pmol/l) } & \multicolumn{4}{|c|}{ Glucose $(\mathrm{mmol} / \mathrm{l})$} & \multicolumn{4}{|c|}{$\mathrm{FFA}(\mathrm{mEq} / l)^{+}$} \\
\hline & (No) & Basal & $0 \mathrm{~min}$ & $90 \mathrm{~min}$ & (No) & Basal & $0 \mathrm{~min}$ & $90 \mathrm{~min}$ & (No) & Basal & $0 \mathrm{~min}$ & $90 \mathrm{~min}$ \\
\hline Diabetic $\$$ & (12) & $58 \pm 6$ & $61 \pm 7$ & $60 \pm 6$ & (12) & $18.7 \pm 1.1$ & $18.6 \pm 0.8$ & $19.0 \pm 1.2$ & $(7)$ & $1.95 \pm 0.42$ & $2.10 \pm 0.37$ & $1.93 \pm 0.29$ \\
\hline Control & (13) & $154 \pm 8$ & $161 \pm 10$ & $162 \pm 7$ & (13) & $5.6 \pm 0.4$ & $5.6 \pm 0.3$ & $6.1 \pm 0.5$ & (8) & $0.53 \pm 0.16$ & $0.50 \pm 0.10$ & $0.57 \pm 0.22$ \\
\hline
\end{tabular}

Values shown are as means \pm SEM. $\S$ : Plasma insulin, glucose and FFA concentrations in diabetic rats were significantly different from those in control rats throughout the study $(P<0.001)$. $^{+}$: FFA levels were measured in the experiments where $3-$ hydroxybutyrate levels were determined in dialysate. 


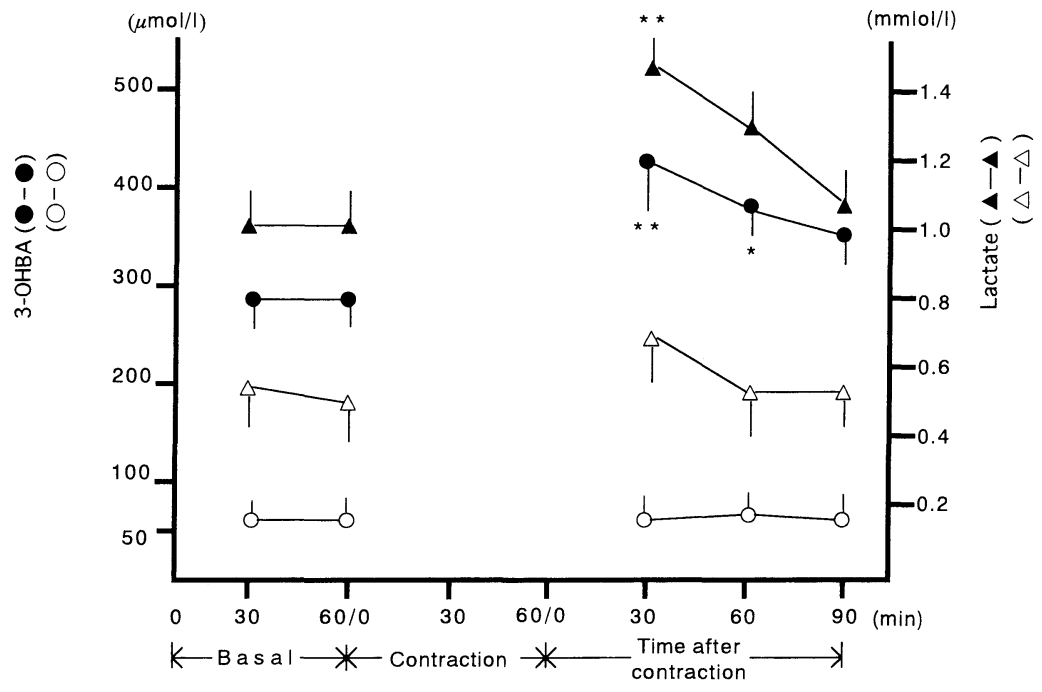

Fig. 1. Changes in 3-hydroxybutyrate (3-OHBA) and lactate concentration in blood dialysate before and after contraction. 3-OHBA concentrations were measured in 7 diabetic $(O)$ and 8 control $(O)$ rats. Lactate concentrations were determined in 5 diabetic $(\boldsymbol{\Delta})$ and 5 control $(\triangle)$ rats. ${ }^{*} P<0.05,{ }^{* *} P<0.01$ vs. basal levels.

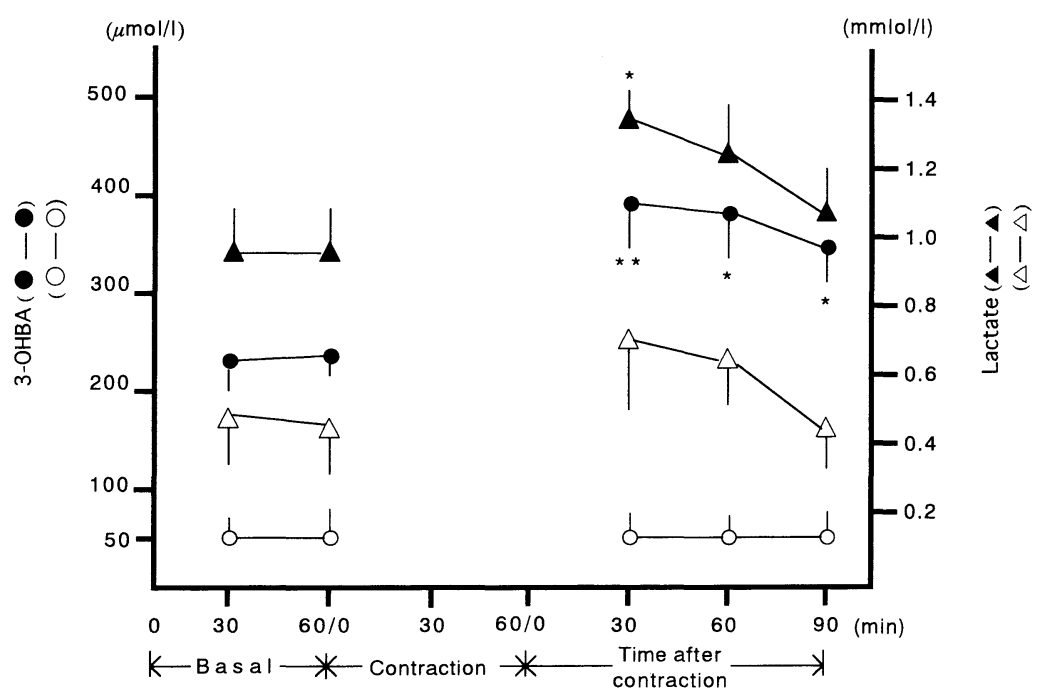

Fig. 2. Changes in 3-hydroxybutyrate (3-OHBA) and lactate concentration in contracting muscle dialysate before, and after contraction. 3-OHBA concentrations were measured in 7 diabetic $(O)$ and 8 control $(\bigcirc)$ rats. Lactate concentrations were determined in 5 diabetic $(\Delta)$ and 5 control $(\triangle)$ rats. ${ }^{*} P<0.05,{ }^{* *} P<0.01$ vs. basal levels.

OHBA concentrations were $240 \pm 15$ and $71 \pm 6$ $\mu \mathrm{mol} / l$ for diabetic and for control rats, respectively (Fig. 5). The lactate concentrations were $1.4 \pm 0.1$ and $0.6 \pm 0.1 \mathrm{mmol} / l$ for diabetic and control rats, respectively (Fig. 6).

\section{Discussion}

The present study demonstrates, with the microdialysis technique, that dialysate 3-OHBA concentrations in contracting and non-contracting 


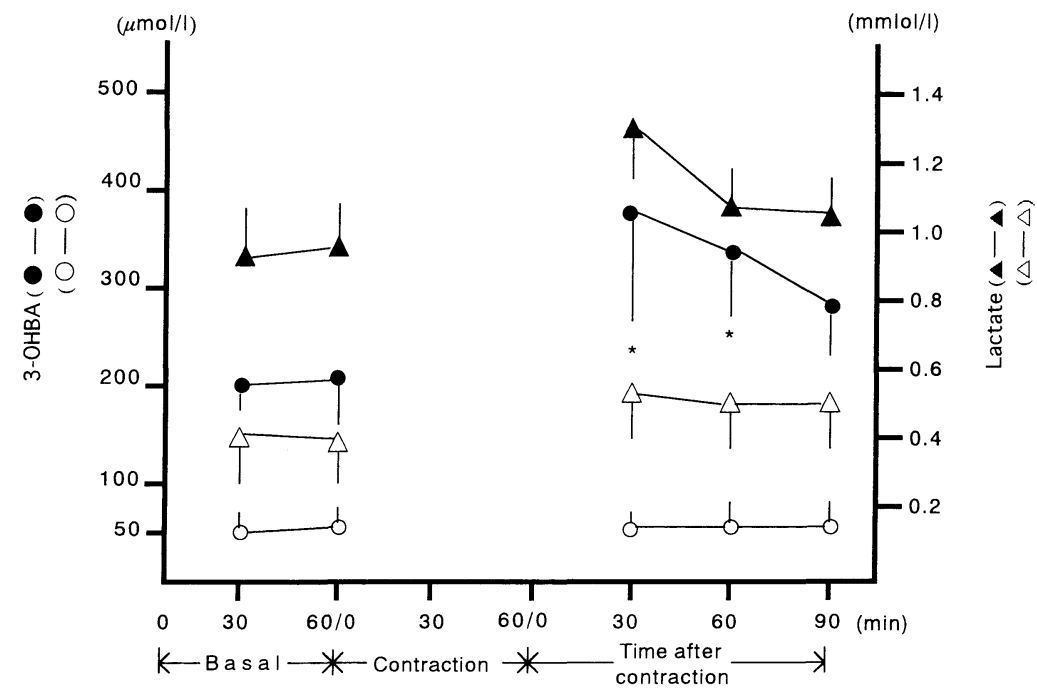

Fig. 3. Changes in 3-hydroxybutyrate (3-OHBA) and lactate concentration in noncontracting muscle dialysate before and after contraction. 3-OHBA concentrations were measured in 7 diabetic $(O)$ and 8 control $(\bigcirc)$ rats. Lactate concentrations were determined in 5 diabetic $(\mathbf{A})$ and 5 control $(\triangle)$ rats. ${ }^{*} P<0.05$ vs. basal levels.

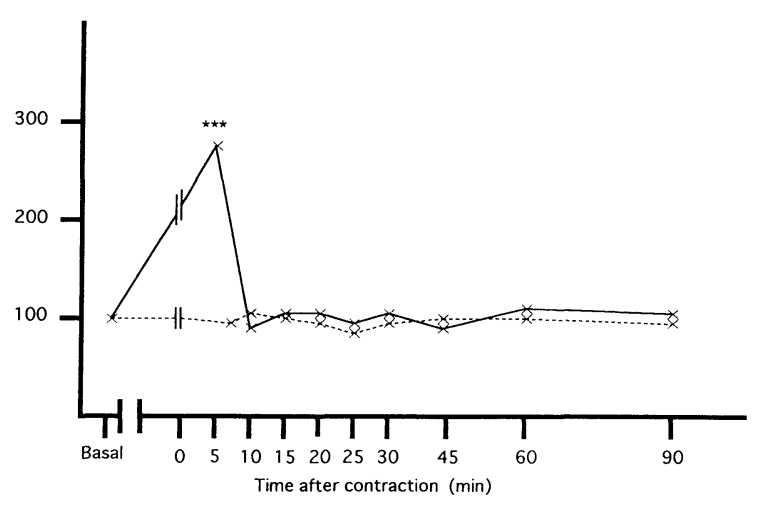

b

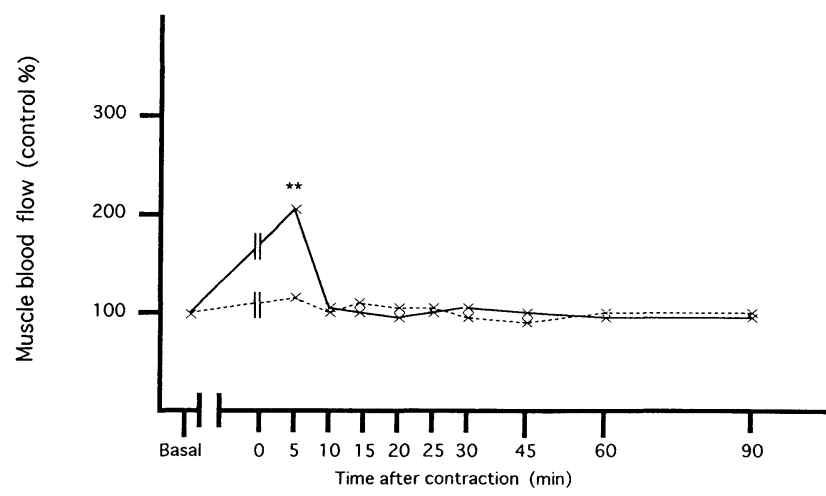

Fig. 4. Blood flow monitoring in contracting $(x-x)$ and noncontracting $(x \cdots \times)$ muscles of diabetic rats (a) and of control rats (b). Changes in blood flow are expressed as a percentage of basal level. Only mean values are shown. ${ }^{* *} P<0.01{ }^{* * *} P<0.001$ vs. basal levels. muscles and in blood after 60 tetani/min contraction were more than $50 \sim 100 \%$ greater in diabetic rats and that this phenomenon was not found in non-diabetic control rats, but dialysate 3OHBA concentrations in contracting and non-contracting muscles and in blood during contraction were not shown in the present study. Dialysate sampling was not performed during contraction since the microdialysis probes were easily moved. There is no doubt that ketone bodies are produced primarily in the liver and transported by the bloodstream to peripheral tissues, where ketone bodies are oxidized, and that, on the other hand, lactate is produced in peripheral tissues and transported to the liver, where it is metabolized. There are two major ketone bodies, 3-OHBA and acetoacetate (AcAc). AcAc is usually at a lower concentration than 3-OHBA and is also unstable. In the present study, 3-OHBA was measured, not AcAc. Although the in vitro recovery of 3-OHBA and lactate remains constant up to at least 800 $\mu \mathrm{mol} / l$ and $3.0 \mathrm{mmol} / l$, respectively, and is useful in describing the diffusion characteristics of the microdialysis probe per se, it is of limited value in predicting the probe behavior in vivo. Thus, the concentration of a substance at the probe outlet is not only determined by the characteristics of the probe membrane itself and the concentration gradient existing across the probe membrane for 


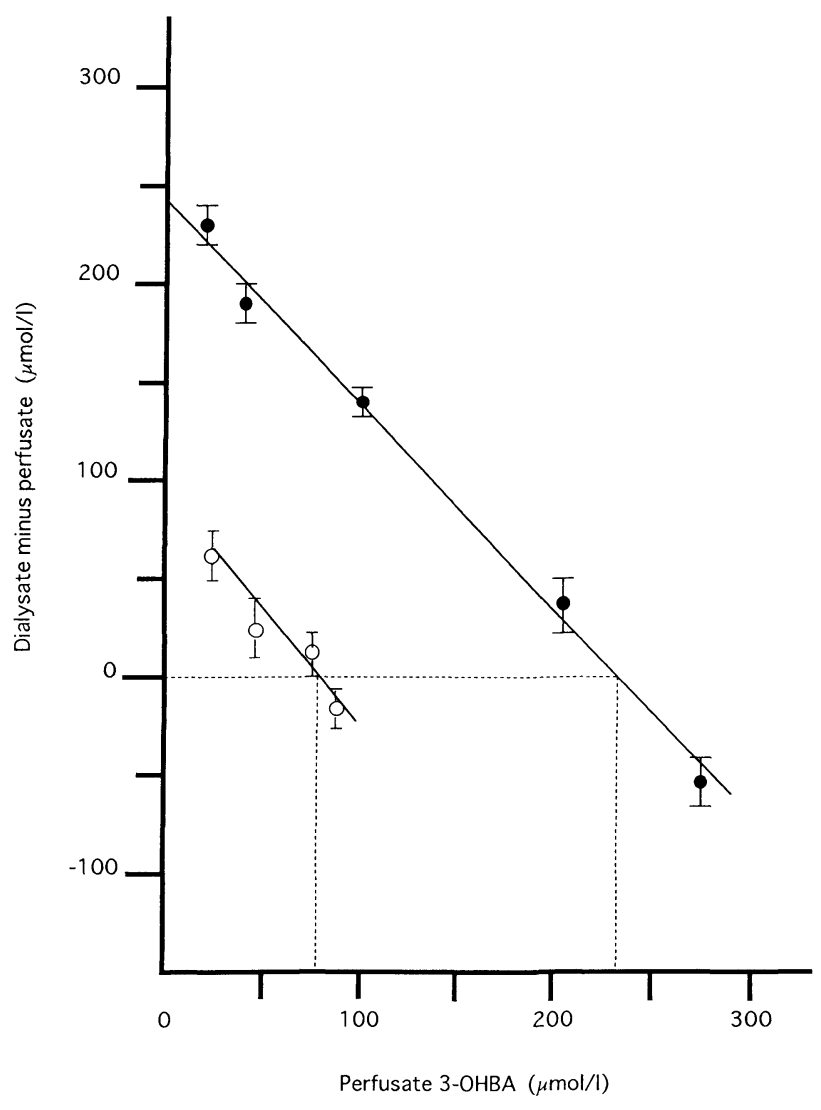

Fig. 5. Relationship between the concentrations of 3hydroxybutyrate ( $3-\mathrm{OHBA}$ ) in perfusate and in dialysate during an equilibrium microdialysis experiment in vivo (-) 5 diabetic, $\bigcirc-\bigcirc ; 5$ control).

that substance, but also is governed by a complex interplay between cellular metabolism and the bloodstream [15]. It is, therefore, important to estimate the absolute intramuscular concentrations of 3-OHBA and lactate in diabetic and control rats by the in vivo calibration method [13] and to monitor the blood flow in contracting and noncontracting muscles [16]. These measurements were carried out in the present study. Blood flow in contracting muscle was significantly increased only for the first $5 \mathrm{~min}$ after contraction in diabetic and control rats. Thereafter it remained constant. Dialysate 3-OHBA concentrations in contracting muscle showed a significant increase in diabetic rats for 90 min after contraction, indicating that 3OHBA removal from contracting muscle was reduced. It is assumed that this phenomenon is mainly due to a decrease in 3-OHBA uptake in contracting muscle. These results are in agreement

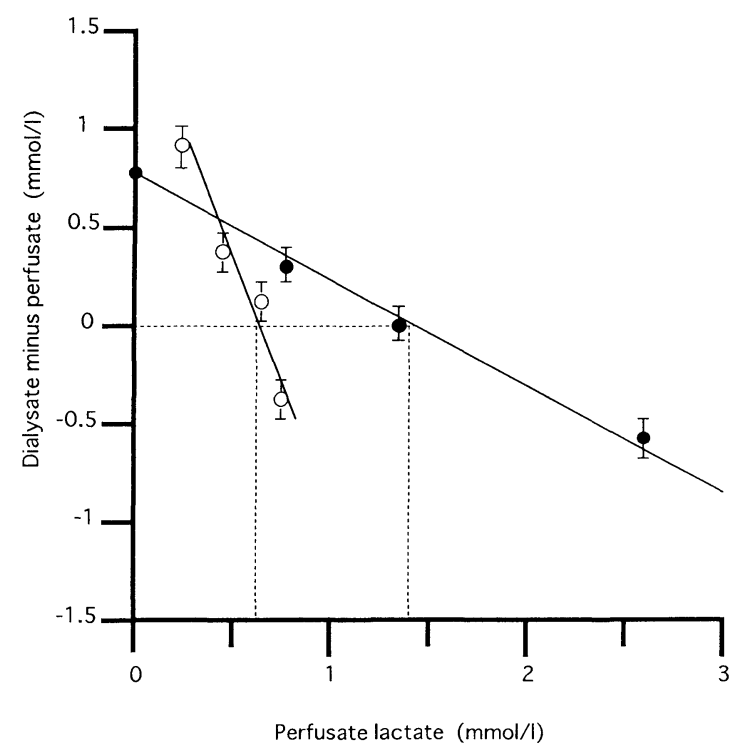

Fig. 6. Relationship between the concentrations of lactate in perfusate and in dialysate during an equilibrium microdialysis experiment in vivo (O- 5 diabetic, $\bigcirc-\bigcirc ; 5$ control).

of the opinion of Féry et al. [9].

It is well known that exercise provokes hepatic ketogenosis in insulin-deprived diabetes [5]. A small amount of plasma insulin can cause inhibition of lipolysis and ketogenesis, which is much more sensitive to insulin than inhibition of hepatic glucose production and stimulation of peripheral glucose uptake [17, 18]. In fact, blood glucose concentrations were kept constant throughout the study. Hepatic ketogenesis is not considered to play a major role in ketosis in the present study. In that case, it is conceivable that reduced 3-OHBA removal in muscle can cause post-exercise hyperketonemiam, but hepatic ketogenesis was not determined. It is possible that hyperketonemia is related to increased hepatic ketone body production. On the other hand, since changes in dialysate 3-OHBA in non-contracting muscle seemed to be identical to those in blood, 3-OHBA in non-contraction muscle might be supplied from the bloodstream. Differences between blood and muscle dialysate concentrations were also found in the present study. These differences may be explained by those in hepatic ketogenesis induced by muscle contraction, but it is unlikely because of the above-mentioned facts. Another explanation is that the differences may be owing to the 
differences in the diffusion properties of blood and muscle.

The utilization of 3-OHBA in muscle requires its mandatory conversion to AcAc, which is associated with reciprocal conversion of NAD to NADH. Wallberg-Henriksson and Holloszy [19] have demonstrated that muscle contraction accelerates glucose transport in insulin-deficient diabetic rat muscle. Insulin-deficiency suppresses glucose oxidation and then increases lactate production via glycolysis, which was found in dialysate after contraction in the present study. Therefore, the impaired capacity to utilize 3-OHBA could occur as a result of a limited tissue availability of NAD [7]. The other mechanism of a decrease in 3-OHBA utilization after contraction in diabetic rats is insulinopenia, which causes a reduction in ketone body oxidation.

Wahren et al. [6, 20], using the femoral or deep forearm vein catheterization techniques in diabetic humans, have shown that ketone body utilization by muscle increases during exercise. Although an increase in intramuscular 3-OHBA concentrations in contracting muscle was found after contraction, and not during contraction, in the present study, their reports seems to be contrary to ours. There are at least two reasons why they came to conclusions opposite ours. First, it is probable that their patients were less completely deprived of insulin than those in the present study, where diabetic rats had not been given insulin since STZ was injected. Second, the intensity and type of exercise in the present study (tetanic contraction at 60 tetani $/ \mathrm{min}$ ) are different from their work (using a bicycle or a hand ergometer at $25 \sim 45 \%$ of the subjects' maximal oxygen uptake).

It is generally stated that diabetic ketosis results from not only an exaggerated stimulation of hepatic ketone body production [6] but also a selective defect of 3-OHBA peripheral utilization [21]. In the present study, the diabetic models were mild, and intramuscular 3-OHBA concentrations during contraction were unknown, but, based upon the results of the present study we conclude that a reduction in 3-OHBA uptake in contracting muscle could play a role in post-exercise hyperketonemia in diabetics.

\section{References}

1. Åstrand P-O (1987) Exercise physiology and its role in disease prevention and in rehabilitation. Arch Phy Med Rehabil 68: 305-309.

2. Yamanouchi $K$, Nakajima $H$, Shinozaki T, Chikada K, Kato K, Oshida Y, Ohsawa I, Sato J, Sato Y, Higuchi M, Kobayashi S (1992) Effects of daily physical activity on insulin action in the elderly. $J$ Appl Physiol 73: 2241-2245.

3. Yamanouchi K, Shinozaki T, Chikada K, Nishikawa T, Ito K, Shimizu S, Ozawa N, Maeno H, Kato K, Oshida Y, Sato Y (1995) Daily walking combined with diet therapy is a useful means for obese NIDDM patients not only to reduce body weight but also to improve insulin sensitivity. Diabetes Care 18: 775-778.

4. Vranic M, Berger M (1979) Exercise and diabetes mellitus. Diabetes 28: 147-163.

5. Berger M, P. Berchtold P, Cüppers HJ, H. Drost H, Kley HK, Müller WA, Wiegelmann W, Zimmermann-Telschow W, Gries FA, Kuüskemper HL, Zimmermann H (1977) Metabolic and hormonal effects of muscular exercise in juvenile type diabetics. Diabetologia 13: 355-365.

6. Wahren J, Sato Y, Östman J, Hagenfeldt L, Felig P (1984) Turnover and splanchnic metabolism of free fatty acids and ketones in insulin-dependent diabetics at rest and in response to exercise. J Clin Invest 73: 1367-1376.

7. Nosadini R, Avogaro A, Trevisan R, Duner E, Marescotti EC, Iori E, Cobeli C, Toffolo G (1985) Acetoacetate and 3-hydroxybutyrate kinetics in obese and insulin-dependent diabetic humans. Am J Physiol 248: R611-R620.

8. Ruderman NB, Goodman MN (1974) Inhibition of muscle utilization during diabetic ketoacidosis. Am J Physiol 226: 136-143.

9. Féry F, de Maerelaer V, Balasse EO (1987) Mechanism of the hyperketonaemic effect of prolonged exercise in insulin-deprived Type I (insulin-dependent) diabetic patients. Diabetologia 30: 298-304.

10. Oshida $\mathrm{Y}$, Yang W-P, Wu W, Ohsawa I, Sato J, Sato $Y$ (1992) Effect of muscle constriction on 3hydroxybutyrate uptake using the microdialysis technique. Horm Metab Res 24: 546-547.

11. Yang W-P, Oshida $Y, W u$ W, Sato J, Ohsawa I, Sato $Y$ (1995) Effect of daily voluntary running on in vivo insulin action in rat skeletal muscle and adipose tissue as determined by the microdialysis technique. Int J Sports Med 16: 99-104. 
12. Shimomura $Y$, Fujii H, Suzuki M, Fujitsuka N, Naoi M, Sugiyama S, Harris RA (1993) Branched-chain 2-oxo acid dehydrogenase complexes activation by tetanic contraction in rat skeletal muscle. Biochemica et Biophysica Acta 1157: 290-296.

13. Lönnroth P, Jansson P-A, Smith U (1987) A microdialysis method allowing characterization of intercellular water space in humans. Am J Physiol 253: E288-E231.

14. Oshida Y, Ohsawa I, Sato J, Sato Y (1993) Effects of adrenodemedullation on in vivo insulin-stimulated glucose utilizationin in relation to glycolysis in rat peripheral tissue. Endocr J 40: 99-106.

15. Bungay PM, Morrison PF, Dedrick RL (1990) Steady-state theory for quantitative microdialysis of solutes and water in vivo and in vitro. Life Sci 46: 105-119.

16. Rosdahl H, Ungerstedt U, Jorfeldt L, Henriksson J (1993) Interstitial glucose and lactate balance in human skeletal muscle and adipose tissue by microdialysis. J Physiol (Lond) 471: 637-657.

17. Groop LC, Bonadonna RC, Del Prato S, Ratheiser
K, Zyck K, DeFronzo RA (1989) Effect of insulin on oxidative and non-oxidative pathway of glucose and FFA metabolism in NIDDM. Evidence for multiple sites of insulin resistance. J Clin Invest 84: 205-213.

18. McGarry JD, Woeltje KF, Kuwajima M, Foster DW (1989) Regulation of ketogenesis and the renaissance of carnitine palmitoyltransferase. Deabetes Metab Rev 5: 271-284.

19. Wallberg-Henriksson H, Holloszy JO (1985) Activation of glucose transport in diabetic muscle: Responses to contraction and insulin. Am J Physiol 249: C233-C237.

20. Hagenfeldt L, Wahren J (1968) Human forearm muscle metabolism during exercise. III. Uptake, release and oxidation of $\beta$-hydroxybutyrate and observations on the $\beta$-hydroxybutyrate/acetoacetate ratio. Scand J Clin Invest 21: 314-332.

21. Sherwin RS, Hendler RG, Felig P (1976) Effect of diabetes mellitus and insulin on the turnover and metabolic response to ketones in man. Diabetes 25: 776-784. 